\title{
Una causa infrecuente de convulsión afebril: gastroenteritis por rotavirus
}

\author{
A rare cause of afebrile convulsion: rotavirus gastroenteritis
}

\author{
Dra. Müsemma Karabel ${ }^{a}$ Dr. Duran Karabel ${ }^{a}$, Dra. Semra Kara ${ }^{a}$, Dra. Tuğba Halıcı Taş ${ }^{a}$ y Dr. Sadi Türkay ${ }^{b}$
}

\begin{abstract}
RESUMEN
La gastroenteritis por rotavirus es la más frecuente de las diarreas infecciosas y ocasiona una importante morbimortalidad en los niños pequeños, además de deshidratación grave y desequilibrios electrolíticos; los signos extraintestinales son infrecuentes. Recientemente se han comunicado convulsiones afebriles asociadas con gastroenteritis por rotavirus, sin encefalopatía, deshidratación, desequilibrio electrolítico o hipoglucemia. Comunicamos el caso de un paciente con convulsiones durante una gastroenteritis por rotavirus, una infección habitual en nuestro país (Turquía), con diversas manifestaciones clínicas, entre ellas, las convulsiones, que pueden verse no sólo en los niños con enfermedades neurológicas o sistémicas sino también en los niños sanos.

Palabras clave: rotavirus, gastroenteritis, convulsión afebril.
\end{abstract}

\section{SUMMARY}

Rotavirus is the most common infectious diarrhea that causes important mortality and morbidities in small children, severe dehydration and electrolyte imbalance. Extraintestinal signs are rare in rotavirus infections. Recently, afebrile seizures associated with rotavirus gastroenteritis but without encephalopathy, dehydration, electrolyte imbalance or hypoglycemia have being reported. In this article, the fact that rotavirus, which is seen commonly in our country, can be confronted with various clinical manifestations was emphasized by reminding that it can be seen not only in infants with neurologic and systemic disease but also in healthy infants.

Key words: rotavirus, gastroenteritis, afebrile convulsion.

http:/ /dx.doi.org/10.5546/aap.2013.e43-e45

\section{INTRODUCCIÓN}

Rotavirus es la causa más común de gastroenteritis aguda, especialmente en invierno en nuestro país (Turquía) y en el mundo. ${ }^{1}$ Se encuentra el antígeno del rotavirus positivo en la materia fecal

a. Department of Pediatrics, Fatih University, Faculty of Medicine, Ankara, Türkiye.

b. Pediatric Cardiology, Fatih University, Faculty of Medicine, Ankara, Türkiye.

Correspondencia:

Dra. Musemma Karabel: musemma.alagoz@gmail.com

Conflicto de intereses: Ninguno que declarar.

Recibido: 26-9-2012

Aceptado: 26-11-2012 entre el $21 \%$ y el $34 \%$ de los pacientes estudiados por gastroenteritis aguda. ${ }^{2}$ Dado que la infección es autolimitada, el tratamiento se basa en el reemplazo de la pérdida de líquido. En estudios recientes, se han comunicado diferentes hallazgos neurológicos presentes durante la gastroenteritis por rotavirus. ${ }^{3}$

Las complicaciones neurológicas no son problemas primordiales en estas infecciones; sin embargo, pueden aparecer convulsiones sin que ocurra deshidratación grave o desequilibrio electrolítico. Los estudios realizados en Asia mostraron que la convulsión afebril asociada con rotavirus es de carácter benigno, ${ }^{4}$ lo cual coincide con los datos provenientes de Europa. ${ }^{5}$ Una publicación reciente mostró que la convulsión asociada a infección por rotavirus es una entidad neurológica relativamente benigna en los niños pequeños. $^{6}$

Presentamos un caso de convulsión afebril durante una gastroenteritis por rotavirus como una manifestación clínica diferente.

\section{CASO CLÍNICO}

Una niña de 22 meses fue atendida en nuestro hospital por una convulsión tónico-clónica, generalizada, de 5 minutos de duración, no acompañada de fiebre. La paciente presentaba vómitos y diarrea maloliente de 2 días de evolución. En el examen físico se encontró una paciente inquieta, con desarrollo normal para su edad. Su temperatura era $36,7^{\circ} \mathrm{C}$, con 128 pulsaciones por minuto, frecuencia respiratoria de 24 por minuto, tensión arterial de 104/46 mmHg. La turgencia cutánea estaba reducida; el tono era normal. No se detectaron alteraciones neurológicas. No había antecedentes en su historia de anomalías congénitas ni enfermedad epiléptica.

Los estudios de laboratorio mostraron: hemoglobina $13 \mathrm{~g} / \mathrm{dl}$, hematocrito $38 \%$, volumen corpuscular medio $78 \mathrm{fL}$, leucocitos $8400 / \mathrm{mm}^{3}$, plaquetas $43700 / \mathrm{mm}^{3}$. El frotis mostró predominio linfocitario y eritrocitos hipocrómicos de tamaño normal.

Otros estudios fueron: $\mathrm{PCR}<1 \mathrm{mg} / \mathrm{dl}$, glucosa $92 \mathrm{mg} / \mathrm{dl}$, urea $17 \mathrm{mg} / \mathrm{dl}$, creatinina $0,3 \mathrm{mg}$ / 
dl, sodio $142 \mathrm{mEq} / \mathrm{L}$, potasio 4,5 $\mathrm{mEq} / \mathrm{L}$, calcio $10,1 \mathrm{mg} / \mathrm{dl}$, fósforo $4 \mathrm{mg} / \mathrm{dl}$, fosfatasa alcalina 182 UI/L, ALT 34 U/L (VN 1-41), AST 15 U/L (VN 1-38).

El LCR fue claro, sin células, con valores normales de proteínas y glucosa. No hubo hallazgos patológicos en la microscopia de la orina ni de la materia fecal. La investigación de antígeno del rotavirus en la materia fecal por el método de aglutinación del látex fue positiva.

A la segunda hora de su hospitalización sufrió una convulsión tónico-clónica que duró 30 segundos. Se le administraron $0,3 \mathrm{mg} / \mathrm{kg}$ de diazepam intravenoso. El EEG fue normal. En el coprocultivo no se aislaron gérmenes.

No hubo hallazgos anormales en la resonancia magnética cerebral realizada luego del egreso del hospital.

\section{DISCUSIÓN}

La deshidratación grave y los desequilibrios electrolíticos pueden provocar convulsiones; $\sin$ embargo, las convulsiones afebriles ocurren en relación con la gastroenteritis por rotavirus sin desequilibrios electrolíticos. Moraka comunicó, en 1982, por primera vez la ocurrencia de esta entidad en Japón. ${ }^{1}$ En la gastroenteritis por rotavirus, el compromiso del sistema nervioso central se ha comunicado en el $2 \%,{ }^{2}$ mientras que las convulsiones febriles y afebriles tuvieron una frecuencia de $6 \%$ a $7 \% .^{7}$

En un estudio sobre gastroenteritis aguda con encefalopatía se detectó la presencia de Shigella sonnei y Salmonella del grupo B en la materia fecal con una frecuencia de $3 \%$; rotavirus fue detectado en $5,7 \%$ y en $2,5 \%$ de los casos no se pudo aislar ningún agente. ${ }^{2,8}$ Narchi y cols. ${ }^{9}$ aislaron rotavirus en 7 casos, en una serie de 14 pacientes con convulsiones afebriles asociadas a gastroenteritis virales, con deshidratación leve, sin antecedentes de convulsiones ni desequilibrios electrolíticos, con crecimiento normal durante el seguimiento de 31 meses y sin repetición de las convulsiones.

Las convulsiones asociadas a infección por rotavirus son casi siempre breves. Sin embargo, algunas pueden perdurar y ser potencialmente mortales, como las que duran más de 5 minutos o el estado epiléptico. ${ }^{6}$ Las convulsiones ocurren habitualmente en el tercer día de enfermedad o después, ${ }^{10}$ como en el caso analizado. Es poco frecuente que los niños con convulsiones asociadas a rotavirus necesiten tratamiento anticonvulsivo prolongado. En este paciente no fue necesario.

En un estudio reciente, se estudió con imáge- nes a niños con convulsiones afebriles con mayor frecuencia que a aquellos con convulsiones febriles. La evaluación con tomografía computarizada es de poca utilidad en las convulsiones asociadas a rotavirus. ${ }^{6,11}$ Los estudios de resonancia magnética fueron normales en ambas oportunidades en el paciente del caso analizado.

Las convulsiones durante la infección por rotavirus pueden ser ocasionadas por fiebre, desequilibrio electrolítico o administración de líquidos de hidratación. Sin embargo, se han descrito niños con infecciones por rotavirus y convulsiones, sin factores desencadenantes. ${ }^{6}$ Algunos estudios sugieren que las convulsiones por rotavirus son inducidas por efecto directo sobre la excitabilidad neuronal. El ácido nucleico del rotavirus y la inmunoglobulina $\mathrm{G}$ específica para rotavirus se han detectado en el líquido cefalorraquídeo de niños con convulsiones afebriles, confirmando que pueden invadir el sistema nervioso central. ${ }^{6,12}$

En estudios recientes sobre la patogenia de la convulsión, se ha discutido el papel del óxido nítrico. Este se halló significativamente elevado en la sangre y el líquido cefalorraquídeo de los pacientes con gastroenteritis por rotavirus en comparación con casos de meningitis purulenta, encefalitis y otras causas de convulsiones afebriles; se ha propuesto que estos niveles altos de óxido nítrico pueden asociarse con las convulsiones directa o indirectamente. ${ }^{13}$

Las convulsiones afebriles relacionadas con gastroenteritis por rotavirus se han considerado una entidad bien definida. ${ }^{9,14}$

La definición de Komori ${ }^{15}$ es la siguiente:

1. Convulsiones generalizadas tónico-clónicas de corta duración, que aparecen entre el primero y el quinto día en el curso de una gastroenteritis, generalmente en los meses de invierno, en niños de 6 meses y 3 años, antes sanos.

2. Deshidratación leve $(<5 \%)$.

3. Tendencia a la recurrencia luego de la convulsión.

4. Electroencefalograma normal en el período interictal.

5. Detección del antígeno del rotavirus en la materia fecal.

6. Resultados normales de los estudios de laboratorio (líquido cefalorraquídeo, electrolitos séricos, glucemia).

7. Generalmente son de buen pronóstico.

El paciente del caso analizado reunía los criterios mencionados: presentó dos convulsiones en el segundo y el tercer día de su enfermedad, tenía 
14 meses, deshidratación ligera, hallazgos normales en el electroencefalograma y en los estudios de laboratorio, y hallazgo de antígeno de rotavirus en la materia fecal.

Nuestro objetivo fue enfatizar que rotavirus se asocia a diferentes manifestaciones clínicas y que puede ocasionar convulsiones no solamente en los niños con enfermedades neurológicas y sistémicas, sino también en los niños sanos. La evaluación y el tratamiento de los niños con convulsiones durante una infección comprobada de esta etiología, pueden ser razonablemente conservadores.

\section{BIBLIOGRAFÍA}

1. Morooka K. Convulsions and mild diarrhea. Shonika (Tokуо) 1982;35:2855-9.

2. Bulut $Y$, İșeri L, Ağel E, Durmaz B. Rotavirus positivities in children with acute gastroenteritis. Inönü University School of Medicine Journal (in Turkish) 2003;10(3):143-5.

3. Kawano G, Oshige K, Syutou S, Koteda Y, et al. Benign infantile convulsions associated with mild gastroenteritis: a retrospective study of 39 cases including virological tests and efficacy of anticonvulsants. Brain Dev 2007;29:617-22.

4. Ben-Ami T, Sinai L, Granot E. Afebrile seizures and rotavirus gastroenteritis: an infrequently recognized association. Clin Pediatr (Phila) 2007;46:178-80.
5. Schumacher RF, Forster J. The CNS symptoms of rotavirus infections under the age of two. Clin Pediatr 1999;211:61-4.

6. Lloyd MB, Lloyd JC, Gesteland PH, Bale JF Jr. Rotavirus gastroenteritis and seizures in young children. Pediatr Neurol 2010;42(6):404-8.

7. Chen HJ, Chen BS, Wang SF, Lai MH. Rotavirus gastroenteritis in children: a clinical study of 125 patients in HsinTien area. Acta Paediatr Sin 1991;32:73-8.

8. Wong V. Acute gastroenteritis-related encephalopathy. J Child Neurol 2001;16:906-10.

9. Narchi H. Benign afebrile cluster convulsions with gastroenteritis: an observational study. BMC Pediatr [serial online] 2004;4:2.

10. Bass ES, Pappano DA, Humiston SG. Rotavirus. Pediatr Rev 2007;28:183-91.

11. World Health Organization. Rotavirus vaccines. Wkly Epidemiol Rec 2007;82:285-95.

12. Hongou K, Konishi T, Yagi S, Araki K, Miyawaki T. Rotavirus encephalitis mimicking afebrile benign convulsions in infants. Pediatr Neurol 1998;18:354-7.

13. Kawashima $H$, Inage $Y$, Ogihara M. Serum and cerebrospinal fluid nitrite/nitrate levels in patients with rotavirus gastroenteritis induced convulsions. Life Sci 2004;74:1397-405.

14. Contino MF, Lebby T, Areinue EL. Rotaviral gastrointestinal infection causing afebrile seizures in infancy and childhood. Am J Emerg Med 1994;12:94-95.

15. Komori H, Wada M, Eto M, Oki H,Aida K, Fujimoto T. Benign convulsions with mild gastroenteritis: a report of 10 recent cases detailing clinical varieties. Brain Dev 1995;17:334-7. 